\title{
Modeling the Adaptive Role of Negative Signaling in Honey Bee Intraspecific Competition
}

\author{
Brian R. Johnson • James C. Nieh
}

Revised: 5 August 2010 / Accepted: 16 August 2010 /

Published online: 14 September 2010

(C) The Author(s) 2010. This article is published with open access at Springerlink.com

\begin{abstract}
Collective decision making in the social insects often proceeds via feedback cycles based on positive signaling. Negative signals have, however, been found in a few contexts in which costs exist for paying attention to no longer useful information. Here we incorporate new research on the specificity and context of the negative stop signal into an agent based model of honey bee foraging to explore the adaptive basis of negative signaling in the dance language. Our work suggests that the stop signal, by acting as a counterbalance to the waggle dance, allows colonies to rapidly shut down attacks on other colonies. This could be a key adaptation, as the costs of attacking a colony strong enough to defend itself are significant.
\end{abstract}

Keywords Animal communication - collective decision making · social insects · honey bees $\cdot$ task allocation

\section{Introduction}

Feedback cycles are key to biological processes ranging from the control of gene transcription, to maintaining physiological homeostasis, to pattern formation and collective decision making in animal societies (Kitano 2002; Franks et al. 2002;

Electronic supplementary material The online version of this article (doi:10.1007/s10905-010-9229-5) contains supplementary material, which is available to authorized users.

B. R. Johnson $(\bowtie)$

Department of Environmental Science, Policy \& Management, University of California, Berkeley, 245 Hilgard Hall, MC3114, Berkeley, CA 94720-3114, USA

e-mail: bjohnson741@hotmail.com

J. C. Nieh

Section of Ecology, Behaviour, and Evolution, Division of Biological Sciences,

University of California, San Diego, 9500 Gilman Dr, MC 0116, La Jolla, CA 92093-0116, USA 
Kholodenko 2006; Sumpter 2006; Detrain and Deneubourg 2006; Marshall and Franks 2009; Becker et al. 2010). Although positive and negative signaling can work together in such processes, most are thought to be based on positive signaling alone. The dance language of the honey bees provides a classic example (Seeley 1995; Passino and Seeley 2006). Foragers who find a rich food source inform their nestmates of its location with the waggle dance (reviewed in Seeley 1989, 1995; Passino and Seeley 2006). This signal leads to a rapid build up of new individuals visiting the food resource, as new recruits subsequently dance themselves. Positive feedback ceases and new recruitment stops, when the food source becomes depleted and recruits cease dancing on their return to the nest (von Frisch 1967; Seeley 1995). The waggle dance signal is thus capable of allocating workers to flower patches without the aid of a negative signal to counterbalance its effects. The honey bee, however, does have a negative signal, the stop signal, which causes waggle dancing bees to stop dancing (Kirchner 1993; Nieh 1993; Pastor and Seeley 2005; Lau and Nieh 2010; Nieh 2010). Because a signal to reduce recruitment seemed unnecessary, the role of the stop signal, within the complex syntax of the dance language, was a mystery, until recently (Nieh 2010).

Whereas positive feedback signals lead to the performance of a behavior, negative signals lead to the cessation of a behavior (Robinson et al. 2005, 2008). In the pharaoh's ant, a negative trail pheromone alerts foragers that a trail is old and probably does not contain currently useful information (Robinson et al. 2005, 2008). Recently, it has been shown that the honey bee stop signal can provide negative feedback in a natural context, fighting over a resource. In short, bees that lose fights perform large numbers of stop signals directed at bees foraging at the same site (thus turning off recruitment to that site), using site odor brought back on foragers' bodies to identify dancers for the same site (Nieh 2010). These studies have focused on aggression between colonies at feeders. A feeder is generally a jar of sugar solution (often as calorically rich as honey) arranged such that a large number of bees can feed from it ad libitum. The only natural context in which bees are able to feed in such a way is either robbing another colony or fighting between colonies over a dying colony. Fighting over dying colonies, which die from the plethora of diseases known to affect Apis mellifera (Winston 1987; Schmid-Hempel 1998; Tarpy and Seeley 2006), is thought to be common and critical for the spread of many diseases. Hence, it is reasonable to assume that bees are making use of behavior that has evolved in the context of intraspecific competition when more than one colony fights over a feeder.

Although it has been understudied relative to intraspecific aggressive behavior in ants, honey bees commonly engage in colony level fights with their neighbors that can lead to the death of weak colonies (reviewed in Winston 1987; Butler and Free 1952). As Seeley (1985) showed, honey bees in the temperate zone only spend a minority of the summer foraging for nectar. The rest of the time, few flowers are blooming. Bee keepers typically refer to such dearth periods as robbing seasons, when bees fight with one another over the large stockpiles of honey each has stored (Butler and Free 1952; Seeley 1985; Winston 1987). Characteristic flight behavior by robbers and intense guarding by defenders has been described (Butler and Free 1952; Winston 1987), but little experimental work has been conducted as researchers are loathe to start their bees fighting, as it 
can get out of control in the unnaturally high population densities typical of apiaries.

Here we explore how the recent results on the specificity and context of the stop signal affect the functioning of the much explored self organizing dance language. Although the stop signal is known to play a role in normal foraging (Pastor and Seeley 2005), we focus on the hypothesis that the stop signal plays a major role in intraspecific competition. At the colony level, the bees have to make a decision regarding the strength of a colony they could attack. At the individual level, the bees have only their experience at the foreign nest. This consists of whether they got honey from the other nest with or without getting into a fight. Thus, for example, if ten bees got honey, with or without getting into fights, but 50 got driven away by guards, with a few killed, then this pattern might indicate that the attacked colony is strong enough to defend itself and the attack should be called off. This could theoretically be done the same way recruitment to a valuable food site is shut down, by simple attenuation, as fewer and fewer bees have success and fewer positive signals (waggle dances) are performed. However, in the context of robbing this could be quite costly because of the mortality of bees sent to the opposing colony. Thus, the stop signal should provide a selective benefit to the colony by counteracting the effect of the waggle dance when there is strong cost (and little benefit) associated with continued waggle dancing. We test this hypothesis with simulations of intraspecific competition using an agent based model of honey bee foraging.

\section{Materials and Methods}

\section{The Model}

Honey bee foraging dynamics have been the subject of continuous study for over 50 years (reviewed in von Frisch 1967; Seeley 1995). Any agent based model of this process will thus be quite elaborate. Figure 1 shows the flow diagram for the present model. This diagram was implemented in the programming language, Netlogo (Wilensky 1999). Essentially, the model builds on the structure of two previous agent based models of honey bee foraging by adding behaviors related to intraspecific fighting and the signal associated with this phenomenon, the stop signal. Readers should consult de Vries and Biesmeijer $(1998,2002)$ for much of the biological justification of the model and for details of many of the behaviors. Given the large number of behaviors simulated, a complete verbal description of the modeling would run into dozens of pages. Thus, researchers interested in minor details of the modeling will need to consult the computer code (available in the Supplemental Information).

All results presented are the means of 50 runs of the simulation model. Error bars were omitted from Figs. 3 and 4 to improve readability.

\section{The Model Environment}

For test bed simulations, we modeled the experiment of Seeley et al. (1991). In this study, a nest and two feeding dishes, $400 \mathrm{~m}$ to the north and south were set up 


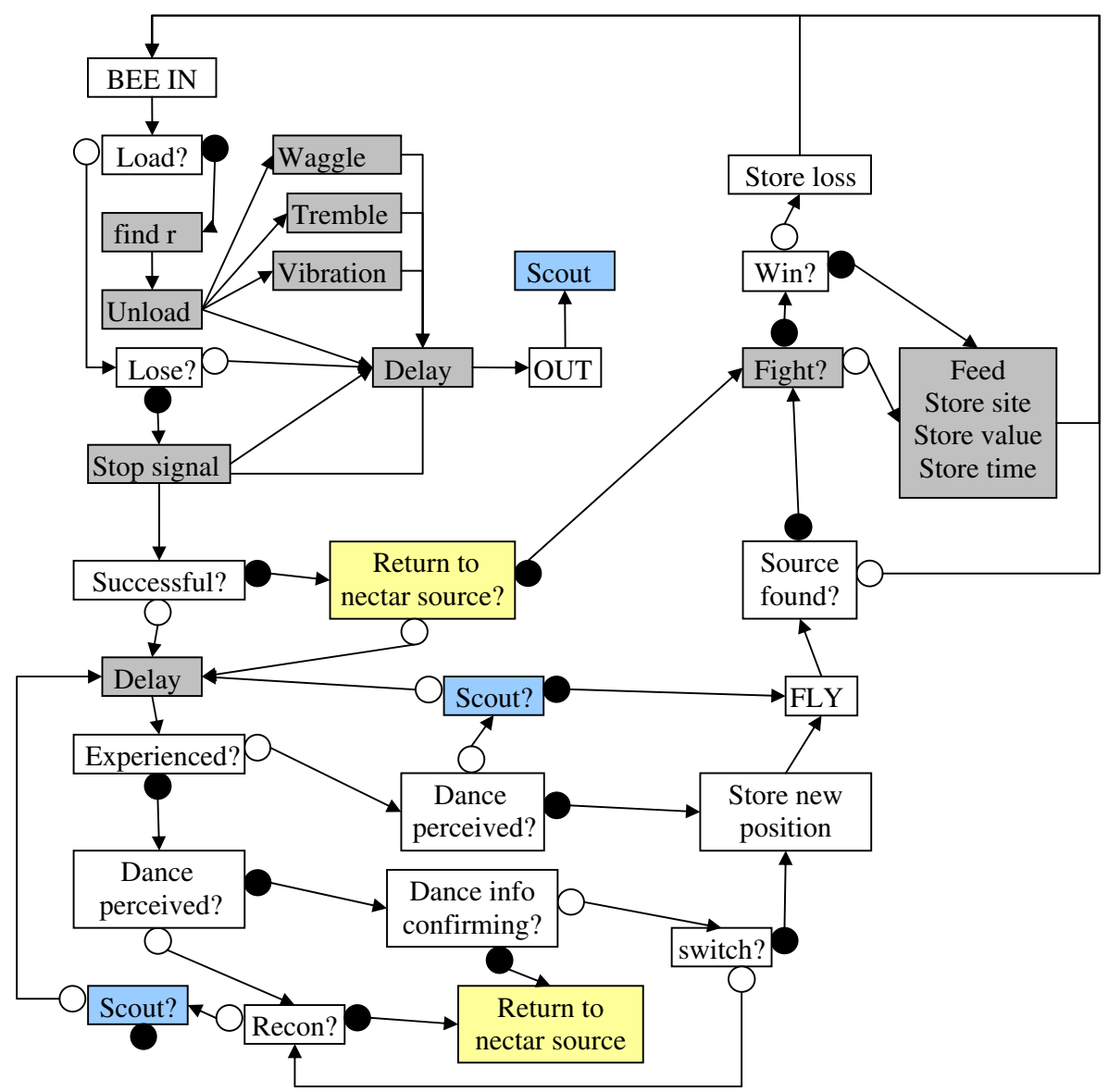

Fig. 1 Flow diagram of the behavioral control structure for foraging honey bees. Grey shading indicates behavioral states, while white shading indicates a decision making procedure. Black bubbles represent, yes, while white bubbles, represent, no. The structure is a simplification of that of de Vries and Biesmeijer (1998). To improve readability some connections are replaced with a color coding pattern to indicate the next transition. After Delay-2, for example, the bee transitions to scouting, which then results in leaving the nest and either finding a site or not. The other two examples of transitioning to scouting are shaded the same color to indicate that their following acts are identical. This choice was made because including lines to the next state in these cases would decrease the readability of the figure. Return to nectar source is treated in the same manner. Find $r$ find receiver

such that one contained high quality sugar solution and the other poor (see Seeley et al. 1991 for details). Midway in the experiment (after 4 h), the solutions were switched. Numbers of recruits arriving at each feeder were recorded. For simulations of intraspecific competition, we used the same environment as for the test bed; however, only one site was used and instead of a feeder we simulated a colony with a variable number of guards. The only part of the nest that is modeled is the dance floor, which is modeled as a square area $40 \times 40$ patches across. Optimization of the movement rate within this dance-floor (based on comparison to experimental results) ensures that the size of this area matches that of a real dance-floor. 


\section{Worker Behavior}

In honey bees, nectar collection is partitioned between two groups of bees: nectar foragers and receivers. Foragers collect nectar in the field, then transfer it near the nest entrance to receivers, who work solely within the nest on processing the nectar into honey and storing it. The model thus has two classes of bees, nectar receivers and foragers. Nectar receivers do not store information; however, foragers can. Nectar receivers move at random on the dance floor until they encounter a forager needing to unload nectar. They then receive nectar and become unavailable for further unloading until they unload the contents of their crop.

By scanning through the sequence of events in Fig. 1 it is possible to trace the trajectory of a forager as it moves through its behavioral repertoire. The reader is referred to de Vries and Biesmeijer (1998) for discussion and review of the data that support these transitions. Essentially, when foragers return to the nest from a food source they may perform one of four signals: the waggle dance, tremble dance, shaking signal, or stop signal depending on their experience within the nest and at the feeder.

\section{Signal Effects}

If an unemployed forager encounters an available waggle dancer, it begins to follow the dance. Only one bee can follow a dance at a time (Judd 1995). A dance takes approximately $5 \mathrm{~s}$ to follow on average (Judd 1995). If the unemployed bee has already visited the source the waggle dancer is advertising, then it is reactivated and returns to it (Seeley 1995). If the novice has not been to the source before, then it learns the location of the food source and attempts to locate it. The tremble dance recruits more nectar receivers (Seeley 1992; Seeley et al. 1996; Thenius et al. 2008). The shaking signal activates bees (Schneider et al. 1986; Nieh 1998; Biesmeijer 2003). In the present model, its function is to increase the size of the forager work force. Foragers perform the stop signal after losing a fight at the feeder and its effect is to stop waggle dancers from dancing (Lau and Nieh 2010; Nieh 2010).

\section{Travel to and from Food Sources}

Foragers that are returning to a food source, simply go straight there, however, scouts and novice recruits do not meander around searching the modeling environment, as it is computationally demanding and unnecessary. Instead, both classes of individual either find the food source (according to distinct probabilities) or not. Thus, when a novice follows a dance, and finds the source, it following the same algorithm as returning foragers for getting to it. If it does not find the source, then it goes into a lost bee state, in which it remains for the length of time bees are known to search for a new site. Scouting is identical, except the length of time bees spend scouting is different. Scouts that find a valuable foraging site return to the nest with nectar and become experienced foragers. 


\section{Parameterization}

Nectar receivers take 20 min to make a circuit up to the honey storage combs and back (Seeley 1989). If a nectar receiver is unable to find a forager to unload for $120 \mathrm{~min}$, it quits the task and the total number of nectar receivers falls by one. Two of the signals, the waggle dance and the tremble dance, were modeled as per de Vries and Biesmeijer (2002). In short, if the time to unloading was less than $60 \mathrm{~s}$, then bees waggle danced with a probability given by the equation, Prob $($ waggle dance $)=0.0435^{*}($ gain $-\operatorname{cost}) / \cos t-0.74$. Costs refer to the amount of energy used to reach the source and return to the nest, while the benefits relate to the quantity of sugar acquired (see de Vries and Biesmeijer 2002; Seeley 1994 for details on the calculation of these parameters). Length of waggle dancing was based on the same equation. If bees do not waggle dance, and if wait time is greater than $20 \mathrm{~s}$, then the probability of tremble dancing increasing linearly to 1 after $80 \mathrm{~s}$. Tremble dancers danced for $60 \mathrm{~s}$ on average (Nieh 1993). Foragers performed the shaking signal with $10 \%$ probability on their first 2 trips to a food source (Schneider and Lewis 2004). Foragers perform the stop signal after losing a fight at the feeder (Lau and Nieh 2010; Nieh 2010). Novice foragers have a 25\% chance of finding a food source (Seeley et al. 1991). Each tremble dancer recruits a finite number of receivers ( 2 for simulations shown in the text) per dance bout, which lasts 120 s (Nieh 1993). Each forager sending shaking signals recruits two new foragers. Waggle dancers that receive a stop signal have a $20 \%$ chance of stopping dancing (Nieh 1993).

\section{Results}

Test Bed Simulations

Bees fight when flower resources are not available. Thus, we are modeling a situation where most of the colony is inactive and a small number of bees are scouting for food sources. In a given time period, some portion of the scouts will find neighboring colonies and initiate the process of collectively determining whether to attack. Therefore, our test bed simulations focused on 1 day of foraging. Day 2 of Seeley et al.'s (1991) data, can also be generated with the present model, but as the factors involved (worker memory over more than a day and quitting times) are beyond the scope of the present study, we save these results for future work. As per de Vries and Biesmeijer (1998) previous model, we have an set of parameter settings that produces output that matches that of real bees (results shown in Fig. 2). This parameterization is as follows: step length on the dance floor of 0.275 patches (rate of movement of foragers and receivers), total forager population size of 225, initial receiver population of 250 , abandonment probability of 0.075 per trip for the poor quality site, and quitting time per patch of $8 \mathrm{~h}$ (Seeley and Towne 1992). We use these settings in our simulations of the effect of the stop signal on intraspecific competition. 
Fig. 2 Comparison of the model's output (means of 50 simulations) with the day 1 empirical results of Seeley et al. (1991). The model is able to replicate both the build-up of foragers at the first site and switching to the second. The parameter settings that lead to this result are shown in the text

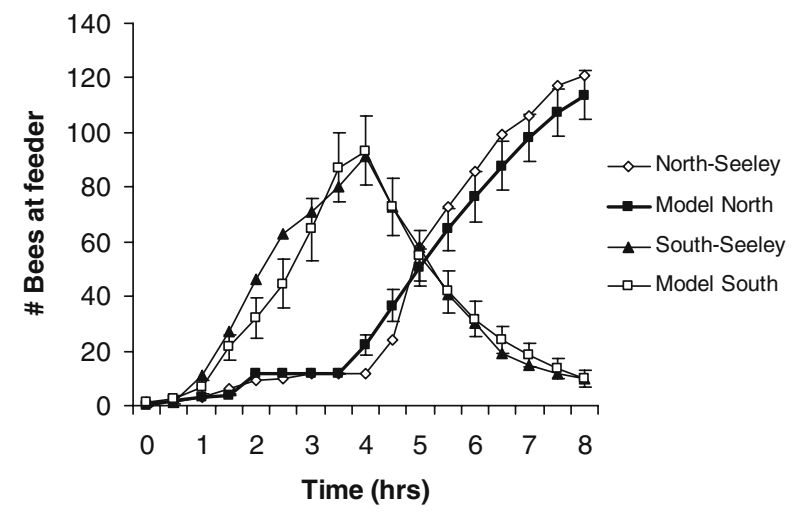

Intraspecific Competition Simulations

To simulate the process of intraspecific competition, we initiated each trial by allowing five bees from the focal colony to find the neighboring colony. We investigated the effect of the stop signal on the dynamics of attack in two contexts: attacks on colonies that can defend themselves, but require a variable amount of time to do so, and attacks on colonies with varying degrees of ability to defend themselves.

Figure 3 explores the role played by the stop signal in attacks on colonies that are strong enough to defend themselves. Specifically, in these simulations the attacked colony ultimately posts enough guards to defend itself (stop 95\% of the bees attacking the nest), but varies in the rate at which they muster defenders. Two sets of simulations were conducted. In the first, the focal (attacking) colony did not use the stop signal, while in the second set of simulations they did. We found that when attacked colonies had a high rate of guard recruitment (rates of guard recruitment shown on Fig. 3), the stop signal had little effect on the attack because most attacking bees encountered guards, lost fights, and hence did not recruit. As the rate of guard recruitment decreased, however, the stop signal had an increasing effect on the rate at which the colony called off the attack. This was because a small number of bees often snuck into the opposing nest no matter how many guards the opposing colony posted. In the absence of the stop signal, these bees consistently performed a small number of recruitment dances, even when it was in the best interest of their colony to call off the attack. When colonies used the stop signal, in contrast, recruitment dances from these rare successful bees were shut down with the result that the colony level attack was abandoned.

Figure 4 explores the role played by the stop signal when attacked colonies vary in their ability to defend themselves. Hence, unlike in Fig. 3, when all attacked colonies defend themselves after a variable period of time, in these simulations some colonies are too weak to defend themselves. Specifically, attacked colonies vary in the ultimate percentage of attacking bees that they can stop at their entrance with recruitment rate of new guards held constant (parameter values shown on Fig. 4). The qualitative dynamics of the attack with and without the stop signal are similar. When attacked colonies are only able to stop a relatively small portion of the attacking bees, the attacking colony quickly recruits more workers for a full attack. When the attacked 
Fig. 3 Effect of the stop signal on the number of foragers attacking another colony with varying rates of guard recruitment in the attacked colony. Results are the means of 50 simulations. All attacked colonies eventually post sufficient guards to defend themselves. At high rates of recruitment, the stop signal has little effect, because most attackers are repelled by guards and do not recruit. As the guard recruitment rates decreases, the effect of the stop signal is more pronounced. As real colonies are slow to recruit guards, the middle and bottom panels are likely to be most relevant for honey bee intraspecific competition
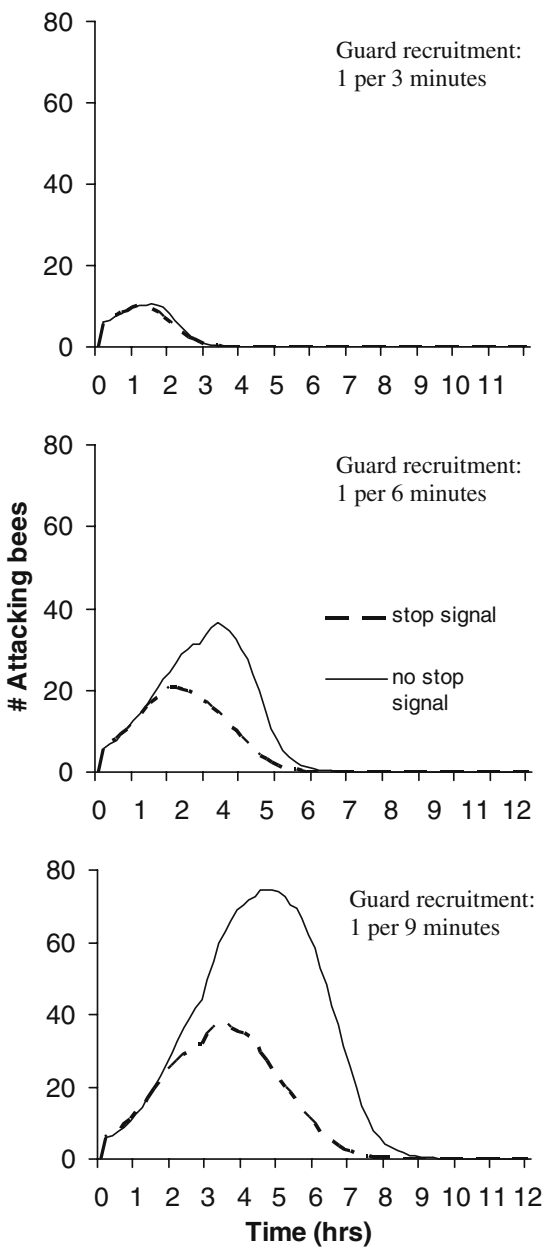

colony is able to stop an intermediate number of attacking workers, the attacking colony continues the attack, but recruitment either builds up slowly or levels off at a stable number of attackers. When the attacked colony is able to stop most of the attacking workers, the attacking colony calls off the attack. In each case, the effect of the stop signal is to decrease the number of attackers the attacked colony must stop to give each respective result. Essentially, attacks are called off at a lower probability of rejection when the attacking colony's foragers are using the stop signal than when they are not. Thus, the effect of the stop signal is to cause the attacking colony to be more conservative in its decisions; that is, they will only attack weak colonies and will quickly call off attacks on colonies able to defend themselves.

\section{Discussion}

Nieh has shown that a rich behavioral repertoire is at play when honeybees fight over nectar resources (Lau and Nieh 2010; Nieh 2010). Bees that win fights recruit 
Fig. 4 Effect of the stop signal on the number of foragers attacking another colony with variation in the total number of guards they are able to post. Results are the means of 50 simulations. The percentage of foragers the attacked colony is maximally able to repel (\% rejection) is shown on each panel. At low rates of rejection, the attacking colony recruits heavily and fully attacks. At intermediate rates of rejection, the number of attacking foragers either builds slowly or levels off, while at high rates of rejection at the foreign colony the attack is called off. In each case, the stop signal decreases the probability required to cause each outcome. The stop signal thus ensures that colonies only fully attack weak neighbors
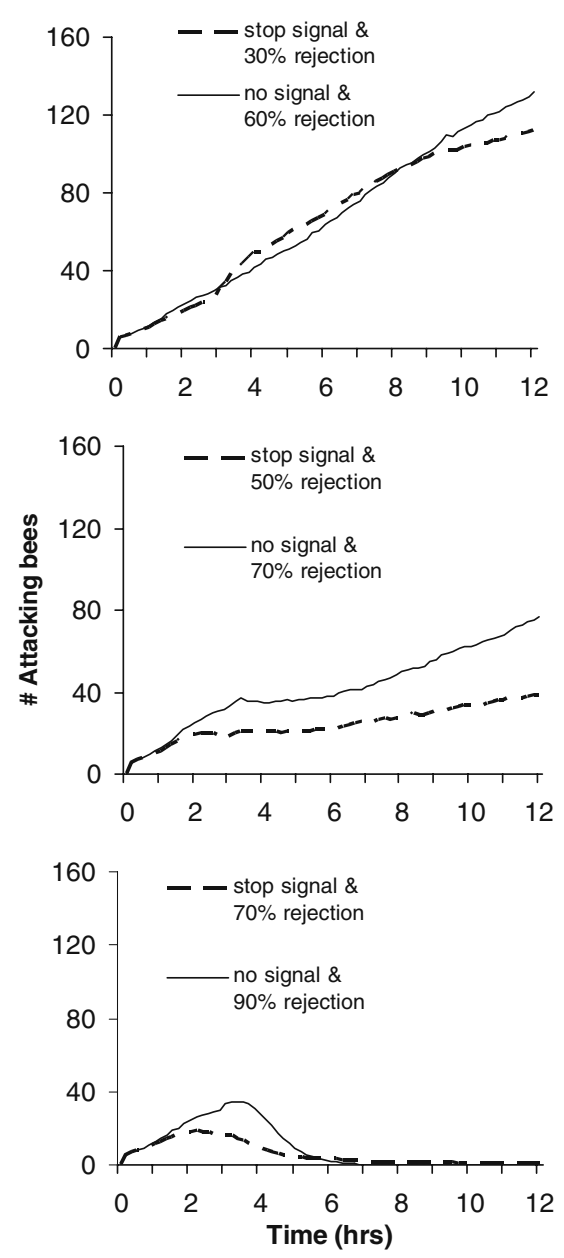

via the waggle dance, while bees that lose fights perform the stop signal (Nieh 2010). This work inspired the present study, which sought to shed light on the natural context in which this behavior may have evolved. We chose the context of intraspecific competition, a phenomena long known to be important for Apis mellifera, but about which little is known. We found that in the context of intraspecific competition, the stop signal can serve as a counterbalance to the rapid recruitment effects of the waggle dance. This result is important because it both increases our understanding of the dance language, one of nature most fascinating systems of communication, and provides a general purpose for negative signaling in other social insects.

Individuals typically make small contributions to collective decision making processes (Deneubourg and Goss 1989; Camazine et al. 2001; Karsai and Balazsi 2002; Merkle and Middendorf 2004; Detrain and Deneubourg 2006; O’Donnell and Bulova 2007; Jeanson et al. 2007; Gove et al. 2009; Johnson 2009a, b). Hence, the cost of a bad decision by an individual is usually small to the colony as a whole. Thus, if a few foragers make the wrong decision to perform waggle dances in the 
context of foraging at flower patches, the cost to the colony might be slight. First, because only a small number of bees are recruited, and second, because the recruits will simply be unsuccessful at finding food at the flower patch and will return home having only wasted a small amount of energy (reviewed in Seeley 1995). In contrast, in the context of between colony fighting, the counterproductive production of waggle dances by even a few bees can have strong costs. First, bees that are successful at invading another colony, acquire fully ripe honey as their reward, meaning they are motivated to produce the maximum number of dance signals when they return to their own nest. Second, when the recruits arrive at the new colony, which may be fully activated for defense, fights occur in which bees can be killed (Butler and Free 1952; Winston 1987). As a forager is able to work for several weeks at a high rate (Schmid-Hempel and Wolf 1988; Visscher and Dukas 1997), the loss of a forager to fighting is far more costly than the benefits of the food gained from a few successful robbing trips. Thus, there should be strong selective pressure for colonies to be both conservative in their decision to attack another colony, and to be able to quickly call off an attack when the costs begin to outweigh the benefits. Theoretically, a negative signal to counterbalance the effects of the waggle dance could solve both of these problems and as the results of this and previous experimental work shows, this is the case. Figure 3 shows that the stop signal allows colonies to quickly call off attacks on colonies able to defend themselves, while Fig. 4 shows that the stop signal makes colonies more hesitant to attack other colonies than they would be otherwise. That colonies have both the capacity for rapid amplification of effort via positive signaling and a capacity for the rapid shutting down of effort via negative signaling makes the honey bee dance language, already one of the most complex animal communication systems known, significantly more complex than previously thought.

With respect to Figs. 3 and 4, the context most relevant to real intraspecific competition is likely Fig. 3. As Downs and Ratnieks (2000) and Couvillon et al. (2008) have shown, honey bees vary in their likelihood of posting guards for defense, such that the number of guards probably increases only in response to direct challenge by other colonies. This makes sense, given that fighting is sporadic and that posting large numbers of guards during periods of no attack would be wasteful. Further, given the study of Couvillon et al. (2008) during which colony level fighting was instigated and the response times of colonies recorded, it is likely that colonies take a significant period of time (many hours) to respond to attack. Hence, attacked colonies with sufficient strength to repel the attack, nevertheless, may exhibit a lag time before they do so. Therefore, once an attacked colony has posted sufficient guards for full defense (and is able to replace them at a rate proportional to mortality), the attacking colony should shut down the attack as quickly as possible, as the cost of lost bees to fighting should strongly outweigh the benefits of lucky robbers. As Fig. 3 shows, the stop signal, particularly if its effect is stronger than the conservative estimate used in the modeling, is an effective method of shutting down attack. Figure 4, however, is probably of less actual biological relevance, although it is useful for understanding the basic function of the stop signal, because colonies unable to post sufficient guards for full defense are likely quite weak (given how easy it is to defend the small nest entrance of a bee colony given the large population size of honey bees) and will be unable to replace the guards they post given even a 
slight rate of guard mortality. In other words, if a colony cannot post the 50 or so guards necessary to defend the entrance, and maintain them, then the top panel of Fig. 4 will inevitably occur, such that a full attack will commence, in which case the stop signal is not relevant.

The results of this study are potentially of relevance for explaining an interesting pattern of intraspecific competition in honeybees. Africanized bees often take over larger colonies of European honeybees (Winston 1987; Pereira and Chaud-Netto 2005). How they do this is a mystery. Although Africanized bees are more aggressive towards humans than European bees, they are actually smaller than European bees and possess no obvious advantage in fighting ability (Schneider et al. 2004). The present study suggests that perhaps the reason European bees are so defenseless towards Africanized bees is because they have a differently tuned intraspecific competition system. Basically, although all Apis mellifera are the same species, there is much local adaptation between bees living in different habitats (Winston 1987; Hepburn and Radloff 1998). The European bees, which live in the temperate zone, which is strongly seasonal, may have recently evolved a defense strategy that assumes that robbing will only occur in times of dearth (Seeley 1985). This is adaptive because during times of plenty in the temperate zone, there is so much nectar available that it would make no sense to fight with other colonies over food. European bees may therefore leave their guard down much of the year and only become defensive in response to the arrival of robbers from other colonies. Africanized bees, however, which are tropical, evolved in a context of much less stability in time and quality of nectar flows (Hepburn and Radloff 1998). Africanized bees may therefore be on the offence and defense year round. Further, because of this generally increased effort towards aggression, the initial attack of Africanized bees may be much stronger than that of European bees with the result that the slow response of European colonies may be insufficient to prevent takeover. Hence, even though a fully alerted European colony may well be able to defend itself, its lack of advance preparation could make it vulnerable to much smaller Africanized colonies. Of course, this is a speculative hypothesis, but hopefully it will encourage future work on this fascinating topic.

Acknowledgements Brian Johnson was supported by an NSF Minority Postdoctoral Fellowship. James Nieh was partially supported through the Opportunities for Research in the Behavioral Sciences program made possible by the NSF Integrative Biology and Neuroscience 0316697 and 0545856 . We thank three anonymous referees for helpful comments on the manuscript. The work reported here complies with the laws of the United States of America.

Open Access This article is distributed under the terms of the Creative Commons Attribution Noncommercial License which permits any noncommercial use, distribution, and reproduction in any medium, provided the original author(s) and source are credited.

\section{References}

Becker MA, Hildenbrandt H, Hemelrijk CK, Moritz RFA (2010) Brood temperature, task division and colony survival in honeybees: a model. Ecol Model 221:769-776

Biesmeijer JC (2003) The occurrence and context of the shaking signal in honey bees (Apis mellifera) exploiting natural food sources. Ethology 109:1009-1020 
Butler CG, Free JB (1952) The behaviour of worker honeybees at the hive entrance. Behaviour 4:262-292

Couvillon MJ, Robinson EJH, Atkinson B, Child L, Dent KR, Ratnieks FLW (2008) En garde: rapid shifts in honeybee, Apis mellifera, guarding behaviour are triggered by onslaught of conspecific intruders. Anim Behav 76:1653-1658

de Vries H, Biesmeijer JC (1998) Modelling collective foraging by means of individual behaviour rules in honey-bees. Behav Ecol Sociobiol 44:109-124

de Vries H, Biesmeijer JC (2002) Self-organization in collective honeybee foraging: emergence of symmetry breaking, cross inhibition and equal harvest-rate distribution. Behav Ecol Sociobiol 51:557-569

Deneubourg JL, Goss S (1989) Collective patterns and decision-making. Ethol Ecol Evol 1:295-311

Detrain C, Deneubourg JL (2006) Self-organized structures in a superorganism: do ants "behave" like molecules? Phys Life Rev 3:162-187

Downs SG, Ratnieks FLW (2000) Adaptive shifts in honey bee (Apis mellifera L.) guarding behavior support predictions of the acceptance threshold model. Behav Ecol 11:326-333

Franks NR, Pratt SC, Mallon EB, Britton NF, Sumpter DJT (2002) Information flow, opinion polling and collective intelligence in house-hunting social insects. Philos Trans R Soc B 357:1567-1583

Gove R, Hayworth M, Chhetri M, Rueppell O (2009) Division of labour and social insect colony performance in relation to task and mating number under two alternative response threshold models. Insect Soc 56:319-331

Hepburn HR, Radloff SE (1998) Honeybees of Africa. Springer, Berlin

Jeanson R, Fewell JH, Gorelick R, Bertram SM (2007) Emergence of increased division of labor as a function of group size. Behav Ecol Sociobiol 62:289-298

Johnson BR (2009a) A self-organizing model for task allocation via frequent task quitting and random walks in the honey bee. Am Nat 174:537-547

Johnson BR (2009b) Pattern formation on the combs of honey bees: increasing fitness by coupling selforganization with templates. Proc R Soc Lond B 276:255-261

Judd TM (1995) The waggle dance of the honey bee: which bees following a dancer successfully acquire the information? J Insect Behav 8:343-354

Karsai I, Balazsi G (2002) Organization of work via a natural substance: regulation of nest construction in social wasps. J Theor Biol 218:549-565

Kholodenko BN (2006) Cell-signalling dynamics in time and space. Nat Rev Mol Cell Biol 7:165-176

Kirchner WH (1993) Vibrational signals in the tremble dance of the honeybee, Apis mellifera. Behav Ecol Sociobiol 33:169-172

Kitano H (2002) Computational systems biology. Nature 420:206-210

Lau CW, Nieh JC (2010) Honey bee stop-signal production: temporal distribution and effect of feeder crowding. Apidologie 41:87-95

Marshall JAR, Franks NR (2009) Colony-level cognition. Curr Biol 19:R395-R396

Merkle D, Middendorf M (2004) Dynamic polyethism and competition for tasks in threshold reinforcement models of social insects. Adapt Behav 12:251-262

Nieh JC (1993) The stop signal of honey bees: reconsidering its message. Behav Ecol Sociobiol 33:51-56

Nieh JC (1998) The honey bee shaking signal: function and design of a modulatory communication signal. Behav Ecol Sociobiol 42:23-36

Nieh JC (2010) A negative feedback signal that is triggered by peril curbs honey bee recruitment. Curr Biol 20:310-315

O'Donnell S, Bulova SJ (2007) Worker connectivity: a review of the design of worker communication systems and their effects on task performance in insect societies. Insect Soc 54:203-210

Passino KM, Seeley TD (2006) Modeling and analysis of nest-site selection by honeybee swarms: the speed and accuracy trade-off. Behav Ecol Sociobiol 59:427-442

Pastor KA, Seeley TD (2005) The brief piping signal of the honey bee: begging call or stop signal? Ethology 111:775-784

Pereira AM, Chaud-Netto J (2005) Africanized honeybees: biological characteristics, urban nesting behavior and accidents caused in Brazilian cities (Hymenoptera: Apidae). Sociobiol 46:535-550

Robinson EJH, Jackson DE, Holcombe M, Ratnieks FLW (2005) Insect communication-'No entry' signal in ant foraging. Nature 438:442-442

Robinson EJH, Ratnieks FLW, Holcombe M (2008) An agent-based model to investigate the roles of attractive and repellent pheromones in ant decision making during foraging. J Theor Biol 255:250-258

Schmid-Hempel P (1998) Parasites in social insects. Princeton University Press, Princeton

Schmid-Hempel P, Wolf T (1988) Foraging effort and life span of workers in a social insect. J Anim Ecol 57:509-521 
Schneider SS, Lewis LA (2004) The vibration signal, modulatory communication and the organization of labor in honey bees, Apis mellifera. Apidologie 35:117-131

Schneider SS, Stamps JA, Gary NE (1986) The vibration dance of the honey bee. 1. Communication regulating foraging on 2 times scales. Anim Behav 34:377-385

Schneider SS, Hoffman GD, Smith DR (2004) The African honey bee: factors contributing to a successful biological invasion. Annu Rev Entomol 49:351-376

Seeley TD (1985) Honey bee ecology. Harvard University Press, Cambridge

Seeley TD (1989) Social foraging in honey bee: how nectar foragers assess their colony nutritional status. Behav Ecol Sociobiol 24:181-199

Seeley TD (1992) The tremble dance of the honey bee: message and meanings. Behav Ecol Sociobiol 31:375-383

Seeley TD (1994) Honey bee foragers as sensory units of their colonies. Behav Ecol Sociobiol 34:51-62

Seeley TD (1995) The wisdom of the hive. Harvard University Press, Cambridge

Seeley TD, Towne WF (1992) Tactics of dance choice in honey bees: do foragers compare dances? Behav Ecol Sociobiol 30:59-69

Seeley TD, Camazine S, Sneyd J (1991) Collective decision-making in honey bees: how colonies choose among nectar sources. Behav Ecol Sociobiol 28:277-290

Seeley TD, Kuhnholz S, Weidenmuller A (1996) The honey bee's tremble dance stimulates additional bees to function as nectar receivers. Behav Ecol Sociobiol 39:419-427

Sumpter DJT (2006) The principles of collective animal behaviour. Philos Trans R Soc B 361:5-22

Tarpy DR, Seeley TD (2006) Lower disease infections in honeybee (Apis mellifera) colonies headed by polyandrous vs monandrous queens. Naturwissenschaften 93:195-199

Thenius R, Schmickl T, Crailsheim K (2008) Optimisation of a honeybee colony's energetics via social learning based on queuing delays. Connect Sci 20:193-210

Visscher PK, Dukas R (1997) Survivorship of foraging honey bees. Insect Soc 44:1-5

von Frisch K (1967) The dance language and orientation of bees. Harvard University Press, Cambridge

Wilensky U (1999) NetLogo. http://ccl.northwestern.edu/netlogo/. Centre for Connected Learning and Computer-Based Modelling, Northwestern University. Evanston, IL

Winston ML (1987) The biology of the honey bee. Harvard University Press, Cambridge 